\title{
Effect of Mineral Fertilization on the Yield of Maize in the Climatic Conditions from ARDS Turda
}

\author{
Susana SFECHIS ${ }^{1}$, Roxana VIDICAN ${ }^{1 *}$, Ioan HAS $^{1,2}$, Mircea IGNEA ${ }^{2}$, Valeria DEAC ${ }^{1,2}$, Ana-Viorica POP ${ }^{3}$ \\ ${ }^{1}$ University of Agricultural Sciences and Veterinary Medicine, Cluj-Napoca, Faculty of Agriculture, Calea \\ Manastur Street, No. 3-5, 400372, Cluj-Napoca, Romania \\ ${ }^{2}$ Agricultural Research-Development Station Turda 27 Agriculturii Street, 401100, Turda, Romania \\ ${ }^{3}$ Department of Food Engineerin ,University of Agricultural Science and Veterinary Medicine, Faculty of \\ Food Science and Technology, Calea Manastur Street, No. 3-5, 400372, Cluj-Napoca, Romania; \\ * corresponding author: roxana.vidican@usamvcluj.ro
}

\begin{abstract}
The importance of the maize fertilization system consists in its directly involvement in the plant growth and development processes conditioning the quantity, quality and economic level of the crops as well as the soil fertility status. Application of unilateral and combined fertilization with different doses of fertilizers and zeolite showed significant variations between experimental variants.
\end{abstract}

Keywords: faeoziom argic, maize, yield, zeolite.

\section{INTRODUCTION}

Maize with a remarkable productive potential among the cereals, is the third important grain crop after wheat and rice and accounts for $4.8 \%$ of the total cropped area and $3.5 \%$ of the value of the agricultural output (Kazem GhassemiGolezani et al., 2011). In the current context of an exponential growth of population, superimposed on maintaining the same level of agricultural lands is necessary to apply effective recipes fertilizers in order to increase production per unit area (Roxana Vidican et. al., 2013).

\section{AIMS AND OBJECTIVES}

The experience aims assessing the of mineral fertilization on maize yield in climatic conditions in year 2013.

\section{MATERIALS AND METHODS}

The experiments were located at ARDS Turda on faeoziom argic soil, following the latin rectangle method with 4 repetitions, 8 variants: $\mathrm{V}_{1}$-control (untreated); $\mathrm{V}_{2}-200 \mathrm{~kg} / \mathrm{ha}$ urea; $\mathrm{V}_{3}-200 \mathrm{~kg} / \mathrm{ha}$ zeolite; $\mathrm{V}_{4}-100 \mathrm{~kg} / \mathrm{ha}$ urea $+100 \mathrm{~kg} / \mathrm{ha}$ zeolite; $\mathrm{V}_{5}-140 \mathrm{~kg} / \mathrm{ha}$ urea+60 $\mathrm{kg} / \mathrm{ha}$ zeolite; $\mathrm{V}_{6}-100$ $\mathrm{kg} / \mathrm{ha} \mathrm{NP}_{20: 20}+100 \mathrm{~kg} / \mathrm{ha}$ zeolite; $\mathrm{V}_{7}-175 \mathrm{~kg} / \mathrm{ha}$ $\mathrm{NP}_{20: 20}+25 \mathrm{~kg} /$ ha zeolite; $\mathrm{V}_{8}-175 \mathrm{~kg} /$ ha zeolite +25

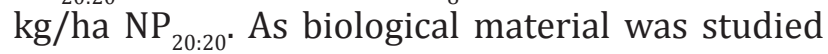
the hybrid Turda Star created at ARDS Turda. Statistical analysis of experimental data was done with statistical software (StatSoft, 2012).

\section{RESULTS AND DISCUSSION}

General evolution of the temperature during vegetation period of the year 2013, followed closely the annual average. There were registered strong deviations from the average amount of rainfall, especially in the second half of the vegetation period (Tab. 1).

Data regarding the production function of different doses mineral fertilizers and their combined zeolite hybrid Turda Star in the climatic conditions of the year 2013, under the influence of climatic factors, there are variations 
Tab.1. The climate in the vegetation period Turda in 2013

\begin{tabular}{cccccccc}
\hline & \multicolumn{7}{c}{ Temperature $\left({ }^{\circ} \mathrm{C}\right)$} \\
\hline Month & $\mathrm{IV}$ & $\mathrm{V}$ & $\mathrm{VI}$ & $\mathrm{VII}$ & VIII & IX & $\mathrm{X}$ \\
\hline Average monthly ${ }^{\circ} \mathrm{C}$ & 12.3 & 16.8 & 19.4 & 20.9 & 22.1 & 13.8 & 11.2 \\
\hline Average 55 years & 9.8 & 14.7 & 17.7 & 19.6 & 19.2 & 14.9 & 9.6 \\
\hline Deviation & +2.5 & +2.1 & +1.7 & +1.3 & +2.9 & -1.1 & +1.6 \\
\hline \multicolumn{7}{c}{ Amount of precipitation $(\mathrm{mm})$} \\
\hline Month & $\mathrm{IV}$ & $\mathrm{V}$ & $\mathrm{VI}$ & $\mathrm{VII}$ & $\mathrm{VIII}$ & $\mathrm{IX}$ & $\mathrm{X}$ \\
\hline Average monthly ${ }^{\circ} \mathrm{C}$ & 53.3 & 79.3 & 86.2 & 37.6 & 44.0 & 57.8 & 67.8 \\
\hline Average 55 years & 44.7 & 67.7 & 84.5 & 76.7 & 55.9 & 40.3 & 32.0 \\
\hline Deviation & +8.6 & +11.6 & +1.7 & -39.1 & -11.9 & +17.5 & +35.8 \\
\hline
\end{tabular}

Tab. 2. Comparative analysis of the evolution production ( $t / h a)$ under the effect of the application of differentiated fertilization

\begin{tabular}{|c|c|c|c|c|c|c|c|c|c|}
\hline Variant & & 1 & 2 & 3 & 4 & 5 & 6 & 7 & 8 \\
\hline & Average & 4.50 & 4.49 & 4.61 & 4.71 & 4.69 & 4.34 & 4.23 & 4.55 \\
\hline 1 & 4.50 & & 0.978 & 0.831 & 0.676 & 0.705 & 0.756 & 0.604 & 0.925 \\
\hline 2 & 4.49 & & & 0.809 & 0.656 & 0.684 & 0.777 & 0.624 & 0.903 \\
\hline 3 & 4.61 & & & & 0.838 & 0.868 & 0.601 & 0.466 & 0.905 \\
\hline 4 & 4.71 & & & & & 0.969 & 0.468 & 0.353 & 0.746 \\
\hline 5 & 4.69 & & & & & & 0.492 & 0.373 & 0.776 \\
\hline 6 & 4.34 & & & & & & & 0.835 & 0.686 \\
\hline 7 & 4.23 & & & & & & & & 0.541 \\
\hline 8 & 4.55 & & & & & & & & \\
\hline
\end{tabular}

$\mathrm{V}_{1}$-control (untreated); $\mathrm{V}_{2}$-urea $200 \mathrm{~kg} / \mathrm{ha} ; \mathrm{V}_{3}$-zeolit $200 \mathrm{~kg} / \mathrm{ha} ; \mathrm{V}_{4}-100 \mathrm{~kg} / \mathrm{ha}$ urea $+100 \mathrm{~kg} / \mathrm{ha}$ zeolite; $\mathrm{V}_{5}-140 \mathrm{~kg} / \mathrm{ha}$ urea $60 \mathrm{~kg} / \mathrm{ha}$ zeolite; $\mathrm{V}_{6}-100 \mathrm{~kg} / \mathrm{ha} \mathrm{NP} \mathrm{20:20}+100 \mathrm{~kg} /$ ha zeolite; $\mathrm{V}_{7}-175 \mathrm{~kg} / \mathrm{ha} \mathrm{NP}{ }_{20: 20}+25 \mathrm{~kg} / \mathrm{ha}$ zeolite; $\mathrm{V}_{8}-175 \mathrm{~kg} / \mathrm{ha}$ zeolite $+25 \mathrm{~kg} / \mathrm{ha} \mathrm{NP_{20:20 }}$ $\mathrm{p}<0.05^{* \circ} \mathrm{p}<0.01^{* * 00} \mathrm{p}<0.001^{* * * 000}$

in production without significant variations compared to the control (Tab. 2).

However fertilized variants revealed significant differences between the variants studied, the highest value was recorded in the variant with $100 \mathrm{~kg} / \mathrm{ha}$ urea+100 kg/ha zeolite (Tab. 2).

\section{CONCLUSION}

In the first experimental year (2013) found no significant differences regarding production increases by applying different doses of mineral fertilizers and zeolite.

Acknowledgement. This paper was published under the frame of European Social Found, Human
Resources Development Operational Programe 2007-2013, project no. POSDRU/159/1.5/ S/132765.

\section{REFERENCES}

1. Kazem Ghassemi-Golezani, Bahareh Dalil, Mohammad Moghaddam, Yaeghoob Raey (2011) Field performance of differentially deteriorated seed lots of maize (Zea mays) under different irrigation treatments Notulae Botanicae Horti Agrobotanici Cluj-Napoca.

2. Vidican Roxana, Mihai Rusu, Ioan Rotar, Marilena Marghitas (2013). User manual application of fertilizers, Risoprint Published, Cluj-Napoca.

3. STATSOFT, INC. (2012) Electronic Statistics Textbook. Tulsa, OK: StatSoft. WEB: http://www.statsoft.com/ textbook/. 